\title{
CONOCIMIENTOS SOBRE LACTANCIA MATERNA EN MADRES PRIMERIZAS. INSTITUTO NACIONAL MATERNO PERINATAL, LIMA 2019
}

\author{
María del Mar Alvarez Lopez ${ }^{1 a}$, Alessandra Patricia Angeles Salcedo ${ }^{1 a}$, Lilian R. Pantoja Sanchez ${ }^{1 \mathrm{bc}}$
}

\begin{abstract}
RESUMEN
Objetivo: Determinar el nivel de conocimiento sobre lactancia materna en madres primerizas del Instituto Nacional Materno Perinatal en Julio 2019. Materiales y métodos: Estudio cuantitativo, descriptivo, transversal. Se encuesto previo consentimiento informado a 276 madres primerizas del INMP usando un cuestionario validado. Resultados: El 80,4 \% de las madres primerizas obtuvo un nivel de conocimiento regular mientras que el $8,3 \%$ obtuvo un nivel de conocimiento malo. El mayor porcentaje de madres con nivel de conocimientos buenos sobre lactancia materna estaba entre los 24 y 29 años $(38,71 \%)$, en cambio, las madres menores o igual a 23 años presentan el mayor porcentaje de nivel de conocimiento malo $(47,82 \%)$. Asimismo, las madres provenientes de provincia representaron el mayor porcentaje de nivel de conocimientos malos $(73,91 \%)$ siendo más del doble de las que procedían de Lima $(26,1 \%)$. Se comprobó que existe una asociación entre la procedencia y la ocupación de la madre con el nivel de conocimiento. Conclusiones: predominó un nivel de conocimiento regular sobre el conocimiento de lactancia materna. En cuanto al perfil de la madre con mal conocimiento fue aquella menor de 23 años, conviviente, procedente de provincia, que trabaja y con estudios secundarios completa; mientras que el perfil de la madre con buen conocimiento fue aquella entre 24 y 29 años, conviviente, procedente de Lima, ama de casa como ocupación y con estudio superior incompleto.
\end{abstract}

Palabras clave: Conocimiento, aprendizaje social, lactancia materna, regular (Fuente: DeCS BIREME).

\section{KNOWLEDGE OF BREASTFEEDING IN FIRST-TIME MOTHERS. INSTITUTO NACIONAL MATERNO PERINATAL, LIMA 2019}

\begin{abstract}
Objective: To determine the level of knowledge on breastfeeding in first-time mothers of the Instituto Nacional Materno Perinatal in July 2019. Materials and methods: Quantitative, descriptive, transversal study. 276 postpartum first-time mothers of INMP were surveyed, with informed consent, using a validated questionnaire. Results: $80.4 \%$ of first-time mothers showed a regular level of knowledge while $8.3 \%$ showed a bad level of knowledge. The highest percentage of mothers with good level of knowledge were between 24 and 29 years old (38.71\%); in contrast, mothers under or equal to 23 years old have the highest percentage of bad knowledge $(47,82 \%)$. In addition, mothers from Province accounted the highest percentage of bad knowledge $(73,91 \%)$, more than double for those who are from Lima $(26,1 \%)$. Also, we found that there is an association between the provenance and occupation of the mother with the level of knowledge. Conclusions: A regular level of knowledge on breastfeeding prevailed. As for the profile of mother with bad knowledge was under 23 years old, domestic partner, from Province, works and full secondary education; while the profile of mother with good knowledge was between 24 and 29, domestic partner, from Lima, housewife and unfinished college.
\end{abstract}

Keywords: Knowledge, social learning, breastfeeding, regular (Source: MeSH NLM).

\section{INTRODUCCIÓN}

La lactancia materna es la forma en la cual un recién nacido se alimenta de la leche de su madre, esta debe ser exclusiva hasta los 6 meses, posterior a este tiempo debe ser complementada con otro tipo de alimentos continuando hasta los dos años o más ${ }^{1}$. La lactancia materna satisface las necesidades nutricionales para el crecimiento y desarrollo del niño, tanto en el ámbito biológico, como psicosocial ${ }^{2,3}$.

Según la OMS "solo un 40,5\% de lactantes menores de seis meses reciben leche materna como alimentación exclusiva"4, Según ENDES, 2018 en nuestro país el $66,4 \%$ de los menores de 6 meses tuvieron lactancia materna exclusiva, mayor en el área rural $(79,6 \%)$ y en las madres que tenían un nivel de educación primario o menor $(80,5 \%)$, sin embargo, en Lima metropolitana solo el $60,4 \%$ de los menores de 6 meses recibieron lactancia materna exclusiva, dato menor al promedio nacional ${ }^{5}$.

El conocimiento sobre la lactancia materna es de suma importancia, no solo para el personal de salud, sino para las madres en general, y abarcan diferentes aspectos, tanto para el recién nacido, como para la madre, en la

Facultad de Medicina Humana Universidad San Martín de Porres, Lima, Perú

Alumno de Medicina,

Médico Pediatra,

Magister en Gerencia de Servicios de Salud

Citar como: Alvarez M, Angeles AP, Pantoja LR. Conocimientos sobre lactancia materna en madres primerizas. Rev Peru Investig Matern Perinat 2020; 9(4):10-15 DOI https://doi.org/10.33421/inmp.2020214

Recibido: 13-11-20 Aprobado: 30-12-20 
que disminuye el riesgo de hemorragia postparto, y por ende disminuye la probabilidad de mortalidad materna ${ }^{6}$, aumenta el periodo de anovulación ${ }^{7}$, menor riesgo de cáncer de ovario y mama ${ }^{8}$ y no representa ningún costo. En el bebé garantiza el crecimiento, inteligencia, desarrollo, fortalece el sistema inmunológico y fortalece el vínculo afectivo entre la madre y el bebé ${ }^{2}$

Existen otros aspectos importantes que la madre debería conocer, como, las medidas de cuidados antes de amamantar; el lavado de las manos ${ }^{9}$, la preparación de sus pechos con masajes circulares etc. ${ }^{10}$, la técnica del amamantamiento, lo que implica la posición correcta del bebé y su madre, la técnica de extracción de la leche materna, la conservación, y la forma de administración a su bebé, esto último crucial en las madres que trabajan fuera de casa, y que están varias horas separadas de sus bebés.

En nuestro país, el nivel de conocimientos en lactancia materna es variado, dependiendo de la metodología usada y de la población estudiada, sin embargo, en investigaciones realizadas en madres primerizas, en diferentes hospitales del país predomina el nivel de conocimientos medio o regular, como lo señala, Vizcarra ${ }^{11}$ (2018), Hospital Regional del Cusco, Villegas y Arroya ${ }^{12}$, Hospital Naylamp, Chiclayo. En este contexto, el presente estudio tiene como objetivo medir el nivel de conocimiento de madres primerizas sobre lactancia materna, en el Instituto Nacional Materno Perinatal, Julio 2019, institución representativa a nivel nacional en la atención materno perinatal en el país.

\section{MATERIALES Y MÉTODOS}

Estudio descriptivo transversal. Se realizó encuestas, a las madres puérperas, primerizas del servicio $E$, del departamento de Obstetricia del Instituto Nacional Materno Perinatal de Lima, nivel III-2, usando un cuestionario validado por (Avendaño y Medrano) ${ }^{13}$, que tiene 19 preguntas, K-R de 0.73 , lo cual indicó que el instrumento cumplía con el requisito de confiabilidad al ser mayor de 0.71.El cuestionario se divide en datos generales de la madre y 14 preguntas sobre conocimientos, por cada acierto se califica con 1 punto, no hay puntos en contra. Se suman los puntajes y se clasifican en: conocimiento bueno (10-14 puntos), conocimiento regular (5-9 puntos) y conocimiento malo (0-4 puntos). Se trabajó con una muestra de 276 madres, calculada con población total de $1,850^{14}$, nivel de confianza $95 \%$, error $5 \%$ y frecuencia esperada de $69,8 \%$ (lactancia materna exclusiva, Encuesta Demográfica y de Salud Familiar 2016 del INEI) $)^{15}$, el muestreo fue por conveniencia, con el criterio de inclusión de madres primerizas y de exclusión madres quechua hablantes.

Se proceso los datos en Excel y se realizó análisis descriptivo con SPSS. Se contó con la autorización del Comité de ética de la Universidad San Martín de Porres y del Instituto Nacional Materno Perinatal, y con el consentimiento informado, firmado por las madres previo a la encuesta.

\section{RESULTADOS}

El promedio de edad de las 276 madres encuestadas, es 23 años, la mayoría conviviente $(75,6 \%)$, ama de casa $(57,6 \%)$, con secundaria completa más del $30 \%$, y más del $50 \%$ proviene de Lima. La mayoría de las madres $(80,4 \%)$ tiene un conocimiento regular sobre lactancia materna, y las madres que tienen un conocimiento bueno $(11,2 \%)$, proporcionalmente son las de 24 a 29 años, que estudian y trabajan, y tienen estudio superior incompleto. (tabla1, 2)

Se realizó análisis de chi cuadrado, encontrando asociación entre el nivel de conocimiento de la madre con la procedencia $(p=0,032)$ y la ocupación $(p=0,005)$.

Tabla 1. Características sociodemográficas de madres primerizas, Instituto Nacional Materno Perinatal, Lima, Julio 2019

\begin{tabular}{|c|c|c|c|c|c|}
\hline Características & $\mathrm{n}=\mathbf{2 7 6}$ & $(\%)$ & Características & $n=276$ & $(\%)$ \\
\hline \multicolumn{3}{|l|}{$\begin{array}{l}\text { Rango de } \\
\text { edades }\end{array}$} & \multicolumn{3}{|l|}{$\begin{array}{l}\text { Nivel de educación } \\
\text { de la madre }\end{array}$} \\
\hline$\leq 23$ años & 153 & $55,4 \%$ & $\begin{array}{l}\text { Primaria } \\
\text { completa }\end{array}$ & 3 & $1,1 \%$ \\
\hline 24-29 años & 87 & $31,5 \%$ & $\begin{array}{l}\text { Secundaria } \\
\text { incompleta }\end{array}$ & 13 & $4,7 \%$ \\
\hline 30-35 años & 30 & $10,9 \%$ & $\begin{array}{l}\text { Secundaria } \\
\text { completa }\end{array}$ & 89 & $32,2 \%$ \\
\hline$\geq 36$ años & 6 & $2,2 \%$ & $\begin{array}{l}\text { Estudio técnico } \\
\text { incompleto }\end{array}$ & 47 & $17,0 \%$ \\
\hline \multicolumn{3}{|c|}{ Procedencia de la madre } & $\begin{array}{l}\text { Estudio técnico } \\
\text { completo }\end{array}$ & 60 & $21,7 \%$ \\
\hline Lima & 143 & $51,8 \%$ & $\begin{array}{l}\text { Estudio superior } \\
\text { incompleto }\end{array}$ & 37 & $13,4 \%$ \\
\hline Provincia & 133 & $48,2 \%$ & $\begin{array}{l}\text { Estudio superior } \\
\text { completo }\end{array}$ & 27 & $9,8 \%$ \\
\hline \multicolumn{3}{|c|}{$\begin{array}{l}\text { Procedencia según } \\
\text { provincia de la madre }\end{array}$} & \multicolumn{3}{|l|}{$\begin{array}{l}\text { Ocupación } \\
\text { de la madre }\end{array}$} \\
\hline Amazonas & 1 & $0,4 \%$ & Estudia & 30 & $10,9 \%$ \\
\hline Ancash & 7 & $2,5 \%$ & Trabaja & 69 & $25,0 \%$ \\
\hline Apurímac & 3 & $1,1 \%$ & Estudia y trabaja & 18 & $6,5 \%$ \\
\hline Arequipa & 2 & $0,7 \%$ & Ama de casa & 159 & $57,6 \%$ \\
\hline Ayacucho & 7 & $2,5 \%$ & & & \\
\hline Cajamarca & 18 & $6,5 \%$ & $\begin{array}{l}\text { Estado civil de } \\
\text { la madre }\end{array}$ & & \\
\hline Cusco & 3 & $1,1 \%$ & Soltera & 45 & $16,3 \%$ \\
\hline Huancavelica & 11 & $4,0 \%$ & Conviviente & 208 & $75,4 \%$ \\
\hline Huánuco & 15 & $5,4 \%$ & Casada & 23 & $8,3 \%$ \\
\hline Ica & 1 & $0,4 \%$ & & & \\
\hline Junín & 17 & $6,2 \%$ & & & \\
\hline La Libertad & 4 & $1,4 \%$ & & & \\
\hline Lambayeque & 8 & $2,9 \%$ & & & \\
\hline Lima & 143 & $51,8 \%$ & & & \\
\hline Loreto & 9 & $3,3 \%$ & & & \\
\hline Madre de Dios & 1 & $0,4 \%$ & & & \\
\hline Moquegua & 1 & $0,4 \%$ & & & \\
\hline Pasco & 3 & $1,1 \%$ & & & \\
\hline Piura & 9 & $3,3 \%$ & & & \\
\hline Puno & 4 & $1,4 \%$ & & & \\
\hline San Martin & 6 & $2,2 \%$ & & & \\
\hline Tacna & 1 & $0,4 \%$ & & & \\
\hline Ucayali & 2 & $0,7 \%$ & & & \\
\hline
\end{tabular}

Fuente: Elaboración propia en base a encuesta en el INMP, 2019 


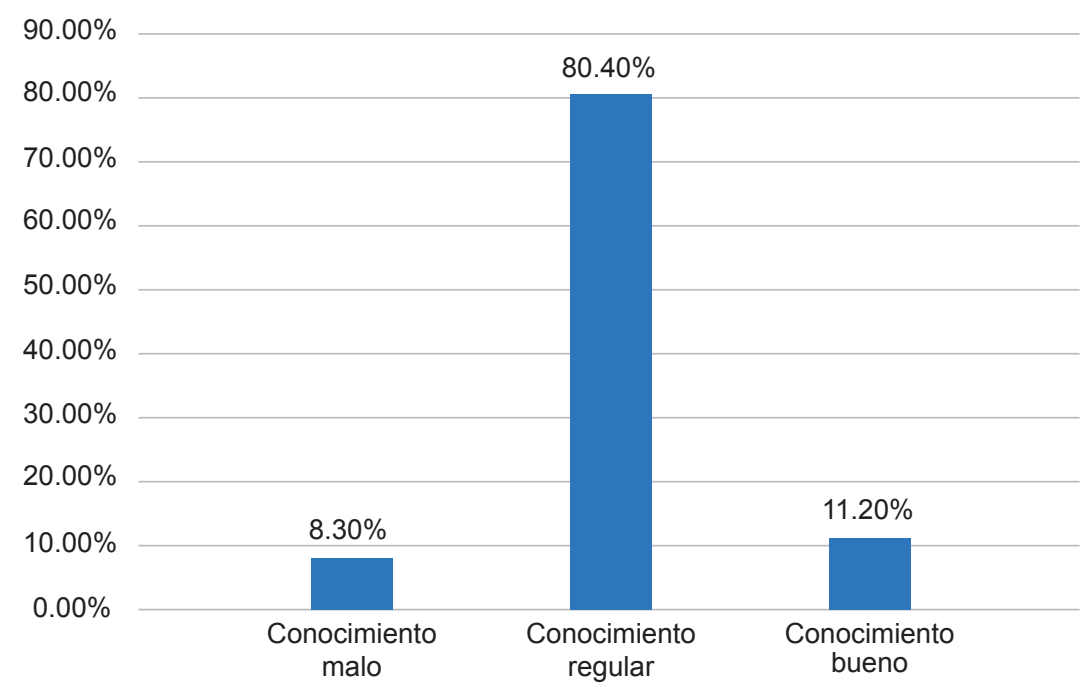

Gráfico 1. Nivel de conocimiento sobre lactancia materna en madres primerizas. Instituto Nacional Materno Perinatal. Lima Julio 2019.

Fuente: Elaboración propia en base a encuesta en el INMP, 2019

Tabla 2. Nivel de conocimiento de las madres primerizas según sus características sociodemográficas, Instituto Nacional Materno Perinatal. Lima Julio 2019.

\section{NIVEL DE CONOCIMIENTO $(n=276)$}

\section{CONOCIMIENTO MALO CONOCIMIENTO REGULAR CONOCIMIENTO BUENO}

\begin{tabular}{|c|c|c|c|c|c|c|}
\hline Rango de edades & $\mathrm{n}$ & $\%$ & $\mathrm{n}$ & $\%$ & $\mathrm{n}$ & $\%$ \\
\hline$\leq 23$ años & 11 & $47,82 \%$ & 131 & $59,01 \%$ & 11 & $35,48 \%$ \\
\hline 24-29 años & 10 & $43,48 \%$ & 65 & $29,28 \%$ & 12 & $38,71 \%$ \\
\hline 30-35 años & 1 & $4,35 \%$ & 22 & $9,91 \%$ & 7 & $22,58 \%$ \\
\hline$\geq 36$ años & 1 & $4,35 \%$ & 4 & $1,80 \%$ & 1 & $3,23 \%$ \\
\hline \multicolumn{7}{|l|}{ Estado civil de las madres } \\
\hline Soltera & 3 & $13,04 \%$ & 35 & $15,77 \%$ & 7 & $22,58 \%$ \\
\hline Conviviente & 19 & $82,61 \%$ & 169 & $76,13 \%$ & 20 & $64,52 \%$ \\
\hline Casada & 1 & $4,35 \%$ & 18 & $8,10 \%$ & 4 & $12,90 \%$ \\
\hline \multicolumn{7}{|l|}{ Procedencia de las madres } \\
\hline Lima & 6 & $26,09 \%$ & 119 & $53,60 \%$ & 18 & $58,06 \%$ \\
\hline Provincia & 17 & $73.91 \%$ & 103 & $46,40 \%$ & 13 & $41,94 \%$ \\
\hline \multicolumn{7}{|l|}{ Ocupación de las madres } \\
\hline Estudia & 2 & $8,70 \%$ & 25 & $11,26 \%$ & 3 & $9,68 \%$ \\
\hline Trabaja & 10 & $43,48 \%$ & 50 & $22,52 \%$ & 9 & $29,03 \%$ \\
\hline Estudia y trabaja & 1 & $4,34 \%$ & 10 & $4,50 \%$ & 7 & $22,58 \%$ \\
\hline Ama de casa & 10 & $43,48 \%$ & 137 & $61,71 \%$ & 12 & $38,71 \%$ \\
\hline \multicolumn{7}{|l|}{ Nivel de educación de las madres } \\
\hline Primaria incompleta & 0 & $0,0 \%$ & 0 & $0,0 \%$ & 0 & $0,0 \%$ \\
\hline Primaria completa & 0 & $0,0 \%$ & 3 & $1,35 \%$ & 0 & $0,0 \%$ \\
\hline Secundaria incompleta & 3 & $13,04 \%$ & 9 & $4,05 \%$ & 1 & $3,23 \%$ \\
\hline Secundaria completa & 8 & $34,78 \%$ & 76 & $34,23 \%$ & 5 & $16,13 \%$ \\
\hline Estudio técnico incompleto & 4 & $17,39 \%$ & 36 & $16,22 \%$ & 7 & $22,58 \%$ \\
\hline Estudio técnico completo & 3 & $13,04 \%$ & 52 & $23,42 \%$ & 5 & $16,13 \%$ \\
\hline Estudio superior incompleto & 2 & $8,70 \%$ & 25 & $11,26 \%$ & 10 & $32,26 \%$ \\
\hline Estudio superior completo & 3 & $13,04 \%$ & 21 & $9,46 \%$ & 3 & $9,68 \%$ \\
\hline
\end{tabular}

Fuente: Elaboración propia en base a encuesta en INMP, 2019 
Tabla 3. Respuestas de las madres primerizas según cada rubro de la encuesta Instituto Nacional Materno Perinatal. Lima Julio 2019.

\begin{tabular}{|c|c|c|c|}
\hline \multicolumn{4}{|c|}{ Repuestas de las madres primerizas según cada rubro de la encuesta } \\
\hline Conocimientos Básicos de Lactancia Materna & & $\mathrm{n}=276$ & $\%$ \\
\hline \multirow[t]{2}{*}{ Respuesta ¿qué es la lactancia materna exclusiva? } & Correcto & 117 & $42,4 \%$ \\
\hline & Incorrecto & 159 & $57,6 \%$ \\
\hline \multirow[t]{2}{*}{ Respuesta ¿hasta cuándo se debe dar de lactar exclusivamente? } & Correcto & 166 & $60,1 \%$ \\
\hline & Incorrecto & 110 & $39,9 \%$ \\
\hline \multicolumn{4}{|l|}{ Conocimientos de la Importancia de la Lactancia Materna } \\
\hline \multirow[t]{2}{*}{ Respuesta ¿cuál es la importancia de la lactancia materna? } & Correcto & 203 & $73,6 \%$ \\
\hline & Incorrecto & 73 & $26,4 \%$ \\
\hline \multicolumn{4}{|l|}{ Conocimientos sobre los Beneficios de la Lactancia Materna } \\
\hline \multirow[t]{2}{*}{ Respuesta ¿cuáles son los beneficios que brinda la lactancia materna a la madre? } & Correcto & 64 & $23,2 \%$ \\
\hline & Incorrecto & 212 & $76,8 \%$ \\
\hline \multirow[t]{2}{*}{ Respuesta ¿cuáles son los beneficios que brinda la lactancia materna al bebé? } & Correcto & 207 & $75,0 \%$ \\
\hline & Incorrecto & 69 & $25,0 \%$ \\
\hline \multicolumn{4}{|l|}{ Conocimientos sobre la Frecuencia de la Lactancia Materna } \\
\hline \multirow[t]{2}{*}{ Respuesta ¿con qué frecuencia se debe dar de lactar a su bebé? } & Correcto & 144 & $52,2 \%$ \\
\hline & Incorrecto & 132 & $47,8 \%$ \\
\hline \multicolumn{4}{|l|}{ Conocimientos del Cuidado de Mamas } \\
\hline \multirow[t]{2}{*}{ Respuesta antes de amamantar a su bebe ¿qué debe realizar primero? } & Correcto & 165 & $59,8 \%$ \\
\hline & Incorrecto & 111 & $40,2 \%$ \\
\hline \multirow[t]{2}{*}{ Respuesta la preparación de la mama para el amamantamiento comprende } & Correcto & 198 & $71,7 \%$ \\
\hline & Incorrecto & 78 & $28,3 \%$ \\
\hline \multicolumn{4}{|l|}{ Conocimientos de Técnica de Amamantamiento } \\
\hline \multirow[t]{2}{*}{ Respuesta la posición de su bebé para dar de amamantar debe ser } & Correcto & 106 & $38,4 \%$ \\
\hline & Incorrecto & 170 & $61,6 \%$ \\
\hline \multirow[t]{2}{*}{ Respuesta ¿cuáles son los signos de succión eficaz? } & Correcto & 87 & $31,5 \%$ \\
\hline & Incorrecto & 189 & $68,5 \%$ \\
\hline \multirow[t]{2}{*}{ Respuesta al terminar el amamantamiento ¿cómo retira la mama? } & Correcto & 15 & $5,4 \%$ \\
\hline & Incorrecto & 261 & $94,6 \%$ \\
\hline \multicolumn{4}{|l|}{ Conocimientos sobre Extracción y Almacenamiento } \\
\hline \multirow[t]{2}{*}{$\begin{array}{l}\text { Respuesta si realiza alguna actividad que no le permite dar de lactar directamente a } \\
\text { su bebe; tendrá que recurrir }\end{array}$} & Correcto & 198 & $71,7 \%$ \\
\hline & Incorrecto & 78 & $28,3 \%$ \\
\hline \multirow[t]{2}{*}{ Respuesta pasos de la extracción de la leche materna } & Correcto & 157 & $56,9 \%$ \\
\hline & Incorrecto & 119 & $43,1 \%$ \\
\hline \multirow[t]{2}{*}{ Respuesta forma de descongelamiento de la leche materna } & Correcto & 172 & $62,3 \%$ \\
\hline & Incorrecto & 104 & $37,7 \%$ \\
\hline
\end{tabular}

Fuente: Elaboración propia 2019

\section{DISCUSIÓN}

Es fundamental que las madres en general, y las madres primerizas en especial tengan conocimiento sobre lactancia materna: beneficios, técnicas de lactancia, recolección y conservación de la leche, ya que esta información la pondrán en practica en beneficio de sus bebes y el de su propia persona. El presente estudio encontró el predominio de un conocimiento "regular" (80,4\% sobre lactancia materna en madres primerizas similar a investigaciones realizados en el país, Vizcarra ${ }^{11}$ (2018), Hospital Regional del Cusco, (53\%) conocimiento regular; Villegas y Arriola ${ }^{12}$ (2018), Hospital Naylamp, Chiclayo,(46,5\%) conocimiento regular; Huaccachi y Yauyo (2016) ${ }^{16}$, Hospital María Auxiliadora, Lima, conocimiento regular (62\%); Ramos $F^{17}$ (2017), Hospital San Juan de Lurigancho, Lima, conocimiento medio $(61,3 \%)$; sin embargo difiere del hallado por Díaz $\mathrm{V}^{18}$ (2018), C.S. Chupaca, Huancayo, en el que la mayoría de las madres primerizas (70\%) tienen conocimiento bajo y de Carmona $^{19}$ (2018), Hospital Regional Las Mercedes, Chiclayo, conocimiento bueno (52\%).

Si consideramos conocimiento regular y malo, como inadecuado el resultado es mayoritario $(88,7 \%)$,similar también al hallado por Vizcarra (82\%), Villegas (46.5), Huaccachi (88\%),datos preocupantes, si tenemos en cuenta que algunos estudios se realizaron en instituciones amigos de la Madre y el niño, tal como lo es el Instituto Materno Perinatal desde Agosto del 2018, sin embargo debemos tener en cuenta que al ser madres primerizas, son las que menos información cuentan, y son el grupo que mas alto $\%$ de conocimiento malo obtiene en las investigaciones ${ }^{20,21}$ .Al ser una población especial, las madres primerizas requieren un mayor apoyo, capacitación y consejería para garantizar un mejor conocimiento de lactancia materna, que se reflejara en una mejor practica con sus bebes. 
Es importante señalar respecto al estudio de Ferro $(2005)^{22}$ realizado hace ya 15 años. en el Instituto Materno Perinatal, en el que las madres primerizas tenían el porcentaje mas alto de nivel de conocimiento malo $(23.5 \%)$, este es menor en nuestros resultados $(8,3 \%)$, aunque las metodologías fueron distintas, esto puede indicar, que en los últimos años, el Instituto Materno Perinatal ha mejorado la educación de las madres en lactancia materna, lo que le permitió certificar como Establecimiento amigo de la madre y el niño, pero que sin embargo aun queda camino por recorrer, ya que el conocimiento bueno $(11,2 \%)$ es bajo.

Respecto a las características sociodemográficas, nuestros resultados son similares al de Carmona ${ }^{19}$, Chiclayo, Ramos $\mathrm{F}^{17}$ Lima, en rango etáreo, nivel educativo y ocupación. Carmona $\mathrm{G}^{19}$ y Quispe D. ${ }^{23}$ encuentran que los conocimientos sobre técnicas de amamantamiento fueron adecuados y de nivel medio respectivamente, en contraste a lo hallado en el presente estudio, en el que el ítem sobre técnicas de amamantamiento, (posición del bebe para lactar, succión eficaz y retiro de la mama) obtuvo el mayor porcentaje de respuestas incorrectas $(66,1 \%)$, mostrando que las madres primerizas del IEMP, requieren mayor educación en aspectos fundamentales, como la técnica de lactancia materna, sin embargo debemos señalar que Carmona estudio la practica de lactancia materna (técnica de lactancia materna), y en nuestro estudio el conocimiento de la técnica de lactancia materna.

Los resultados de conocimientos sobre extracción de lactancia materna $(71.7 \%)$, están ligados a que el INMP cuenta hace varios años con el primer banco de leche humana en el país, lo cual es una fortaleza en la atención de calidad al neonato, no encontramos estudios comparables para el análisis.

Quispe (2015) ${ }^{24}$, Hospital Daniel Alcides Carrión-Lima encontró que $93 \%$ de las madres primíparas encuestadas conocía el concepto de lactancia materna exclusiva, a diferencia de lo hallado en la presente investigación, donde el $57,6 \%$ respondió de forma incorrecta a un concepto básico como es el significado de la lactancia materna exclusiva, proporción muy alta, dada la importancia de la lactancia materna exclusiva para el bebe; sin embargo respecto a la importancia de la leche materna $(73,6 \%)$ es mayor al hallado por Quispe, esto podría ser atribuido a que se esta enfatizando en la educación a las madres en la importancia de la lactancia materna en el desarrollo del recién nacido, y no en los otros aspectos como LME, beneficios de la lactancia materna para la madre y la técnica de lactancia, en los que es necesario, a la luz del presente estudio, que el personal de salud lo realice, para de esta forma fortalecer el INMP como establecimiento amigo de la madre y el niño.

La asociación hallada del nivel de conocimiento con la ocupación de la madre y la procedencia, muestra relación entre variables, que requieren investigaciones posteriores con otra metodología, sin embargo los resultados muestran relación de la ocupación de la madre con el nivel de conocimiento al igual que otras investigaciones ${ }^{25,26}$, y difiere de lo hallado por Ferro ${ }^{22}$ en el INMP, pero ya hace mas de una década, lo que podría ser explicado por la mayor incorporación de las madres al trabajo adicional fuera de casa. ${ }^{27}$

La limitación principal fue el muestreo por conveniencia; además del sesgo del recuerdo de las madres, a pesar de ser madres puérperas, tenían diferentes horas de puerperio.

Consideramos que con los resultados dejamos una referencia sobre el nivel de conocimientos sobre la lactancia materna, tanto en los conceptos básicos de lactancia materna, beneficios binomio madre-hijo, así como técnicas de amamantamiento, almacenamiento y extracción de la leche materna; para que de esta forma el personal de salud pueda diseñar estrategias y programas educativos dirigidos con mayor énfasis en temas donde hay un mayor desconocimiento y así poder reforzarlos para reducir las brechas actuales, en beneficio de los bebes, sus madres y de la sociedad en su conjunto.

Financiamiento: La presente investigación fue autofinanciada por el autor.

Conflicto de intereses: El autor declara no tener ningún conflicto de interés.

\section{REFERENCIAS BIBLIOGRÁFICAS}

1. SOMOS LECHEROS: Lactancia. Ministerio de Salud. (Internet) https://www.minsa.gob.pe/Especial/2017/lactancia/ index.asp. Accedido 31 de agosto de 2019.

2. OMS | 10 datos sobre la lactancia materna». Organización Mundial de la Salud, http://www.who.int/features/factfiles/ breastfeeding/es/. Accedido 31 de agosto de 2019.

3. Alvarez V. S, Orozco M.G. Amamantamiento, aspectos psicosociales, en Revista Mexicana de Pediatría, Vol. 85, No. 6 Noviembre-Diciembre 2018 pp 230-234

4. Organización Mundial de la Salud (OMS). 10 datos sobre la lactancia materna (Internet). Agosto 2017 (citado 16 de agosto 2019). Disponible en: https://www.who.int/features/factfiles/ breastfeeding/es/

5. Encuesta Demográfica y de Salud Familiar - ENDES. Instituto Nacional de Estadística e Informática. Lima, 2019. (citado 31 de Agosto 2019). Disponible en: https://www.inei.gob.pe/ media/MenuRecursivo/publicaciones_digitales/Est/Lib1656/ index1.html

6. Infante T, Almarcha L. Hemorragia postparto y su relación con el tipo de alimentación del recién nacido. España. Mayo, 2016. (Internet) Disponible en:https://www.enfermeriadeciudadreal. com/articulo_imprimir.asp? idarticulo=527\&accion=. Accedido 31 de agosto de 2019.

7. Saadeh R, D. Benbouzid. "Lactancia materna y espaciamiento de los nacimientos: importancia de la obtención de información para las políticas de salud pública." Boletín 
de la Oficina Sanitaria Panamericana (OSP); 111(2), ago. 1991. (Internet) Disponible en: http://iris.paho.org/ xmlui/bitstream/handle/123456789/16578/v111n2p122. pdf?sequence=1\&isAllowed=y. Accedido 31 de agosto de 2019.

8. Organización de las Naciones Unidas para la Alimentación y la Agricultura. FAO: Capitulo 7 Lactancia Materna. (Internet). Nueva York, Estados Unidos: FAO; 2002 [actualizado 9 de Feb 2018; citado 11 de mar 2018]. Disponible en: http://www. fao.org/docrep/006/w0073s/w0073s0b.htm

9. Guía de lactancia materna para profesionales de la salud. Asociación Española de Pediatría. España, 2010. (Internet) Disponible en: https://www.aeped.es/sites/default/files/8guia_prof_la_rioja.pdf. Accedido 31 de agosto de 2019.

10. Manual Lactancia Materna. UNICEF. Ecuador, 2012. Disponible en: https://www.unicef.org/ecuador/Manual_ lactancia_materna_web_1.pdf. Accedido 31 de agosto de 2019.

11. I.Vizcarra. Nivel de conocimiento y su relación con la práctica de lactancia materna exclusiva en madres primíparas, Hospital Regional del Cusco-2018. (Internet) 2019. (citado 20 Sep 2019) Disponible en: http://repositorio.uandina.edu. pe/bitstream/UAC/3314/2/Ingrid_Tesis_bachiller_2019_ Part.1.pdf

12. Villegas Mera, E. V., \& Arriola Arízaga, M. C. (2018). Conocimientos y prácticas sobre Lactancia Materna Exclusiva en madres primíparas que acuden al Servicio de Crecimiento y Desarrollo del Hospital Naylamp, Chiclayo. ACC CIETNA: Revista De La Escuela De Enfermería, 3(2), 19-28. https://doi. org/10.35383/cietna.v3i2.43

13. Avendaño M, Medrano K. Conocimiento y práctica sobre lactancia materna exclusiva que tienen las madres adolescentes primíparas en un hospital nacional-2017. (Internet) 2019. (citado 17 Mayo 2018) Disponible en: http://repositorio.upch.edu.pe/bitstream/handle/ upch/853/Conocimiento_AvendanoSilva_Mirley. pdf?sequence $=3$ \&isAllowed $=y$

14. Instituto Nacional Materno Perinatal. Análisis de la Situación de los Servicios Hospitalarios - ASISHO - INMP- Perú 2016. [Internet]. Lima: Instituto Nacional de Estadísticas e Informática; 2017 [citado 17 Mayo 2018] Disponible en: http:// www.inmp.gob.pe/institucional/asis-ho/1421426743

15. Instituto Nacional de Estadísticas e Informática. Encuesta Demográfica y de Salud Familiar- Perú 2016. [Internet]. Lima: Instituto Nacional de Estadísticas e Informática; 2017 Mayo [citado 17 Mayo 2018] Disponible en:https://www.inei.gob.pe/ media/MenuRecursivo/publicaciones_digitales/Est/Lib1433/ pdf/cap010.pdf

16. Huaccachi F, Yauyo Y. "Conocimientos y prácticas sobre lactancia materna en primíparas, Hospital Nacional María Auxiliadora". [Tesis Pregrado para optar el grado de Lic. en Enfermería]. Universidad Privada Norbert Wiener-2016. Disponible en: https://docplayer.es/26508956-Universidadprivada-norbertwiener.html

17. Ramos F. Nivel de conocimiento, actitudes y practicas sovre lactancia materna exlcusiva en primiparas del hospital San Juan de Lurigancho-2017. Perú. [Citado 13 de oct 2019]. Disponible en: http://repositorio.uwiener. edu.pe/bitstream/handle/123456789/769/TITULO\%20 $\% 20$ Ramos $\% 20$ Tomayro $\% 20$ Flor $\% 20$ De $\% 20$ Maria. pdf? sequence $=1 \&$ isAllowed $=y$

18. Diaz V, Solis A. Conocimientos sobre lactancia materna exclusiva en madres primerizas en el centro de salud Chupaca
2018, Tesis EAP de Enfermería, Universidad Privada de Huancayo Franklin Roosevelt.

19. Carmona G. Nivel de conocimiento y prácticas sobre lactancia materna en madres primerizas del Hospital Regional Docente Las Mercedes- Chiclayo, 2018. [citado 5 de octubre 2019]. Disponible en: http://www.pead.uss.edu.pe/handle/uss/5023

20. Cerdan, Espinoza. Nivel de conocimiento y practicas sobre lactancia materna exclusiva en madres atendidas en el Hospital Simon Bolivar Cajamarca, Peru 2019.

21. Sota L. "Actitudes $Y$ Prácticas De Lactancia Materna Exclusiva En Madres Primerizas Que Acuden Al Hospital Antonio Lorena - Cusco 2017". Tesis de licenciatura. Universidad Andina del Cuzco; 2017. Disponible en: http://repositorio.uandina.edu.pe/ bitstream/UAC/1156/3/Liseth_Tesis_bac hiller_2017.pdf

22. Ferro $\mathrm{M}$, Flores $\mathrm{H}$. Nivel de conocimiento en lactancia materna y su relación con factores socioculturales en puerperas del IEMP, de Octubre a Diciembre del 2005, Tesis, UNMS, Accedido 7/9/20, en https://cybertesis.unmsm.edu.pe/bitstream/ handle/20.500.12672/2975/Ferro_sm.pdf?sequence=1

23. Quispe D. Nivel de conocimiento sobre lactancia materna en primíparas hospitalizadas en el Hospital de Apoyo II-2. 2019. Perú. [Citado 5 de octubre 2019]. Disponible en: http:// repositorio.upao.edu.pe/bitstream/upaorep/4591/1/RE_ MEDP.HUMA_DIANA.QUISPE_LACTANCIA.MATERNA_ DATOS.pdf

24. Quispe L. Conocimiento de la primiparas sobre lactancia materna exclusiva en el servicio de alojamiento conjunto Hospital Nacional Daniel Alcides Carrión. 2015. Perú. [Citado 13 de oct 2019]. Disponible en: http://cybertesis. unmsm.edu.pe/bitstream/handle/cybertesis/8307/Quispe gl. pdf?sequence=1\&isAllowed=y

25. María D. Ramiro González, Honorato Ortiz Marrón, Celina Arana Cañedo-Argüelles, María Jesús Esparza Olcina, Olga Cortés Rico, María Terol Claramonte, María Ordobás Gavín. Prevalencia de la lactancia materna y factores asociados con el inicio y la duración de la lactancia materna exclusiva en la Comunidad de Madrid entre los participantes en el estudio ELOIN. Anales de Pediatría (English Edition), Volume 89, Issue 1, July 2018, Pages 32-43. https://doi.org/10.1016/j. anpedi.2017.09.002

26. Gutiérrez AE, Barbosa D, González R, Martínez O. Nivel de conocimiento sobre la lactancia materna. Rev Cubana Enfermer [Internet]. $2001 \mathrm{Abr}$ [citado 2020 Oct 09]; 17(1): 4246. Disponible en: http://scielo.sld.cu/scielo.php?script=sci_ arttext\&pid=S0864-03192001000100007\&lng=es.

27. Canales DM, Caceres R. Conocimientos, Actitudes y Prácticas sobre Lactancia Materna Exclusiva de las madres de niños menores de seis meses atendidos en el Centro de Salud Leonel Rugama.Estelí. Departamento De Estelí Noviembre 2015. Otra thesis, Universidad Nacional Autónoma de Nicaragua, Managua.

\section{Correspondencia:}

María del Mar Alvarez Lopez.

Dirección: Los Manzanos 130, urbanización Monterrico Sur, La Molina.

Correo: marimar920@hormail.com

Teléfono: 933131030 